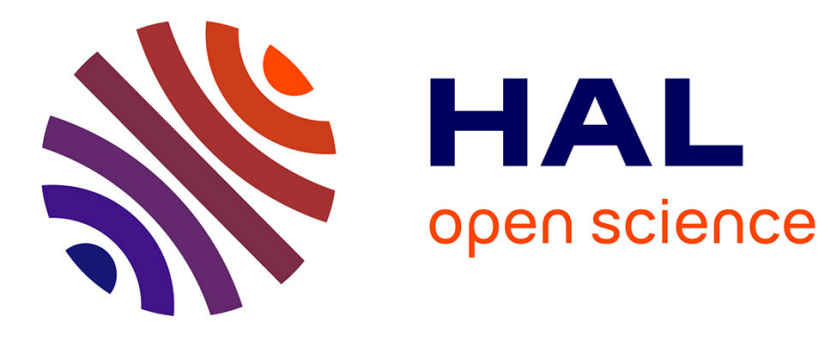

\title{
Hybrid metallic ion-exchanged waveguides for SPR biological sensing
}

\author{
Sandie de Bonnault, Davide Bucci, P. .J. Zermatten, P. Charette, \\ Jean-Emmanuel Broquin
}

\section{To cite this version:}

Sandie de Bonnault, Davide Bucci, P. .J. Zermatten, P. Charette, Jean-Emmanuel Broquin. Hybrid metallic ion-exchanged waveguides for SPR biological sensing. SPIE OPTO, Feb 2015, San Francisco, United States. pp.93650D, 10.1117/12.2077648 . hal-01972286

\section{HAL Id: hal-01972286 https://hal.science/hal-01972286}

Submitted on 6 Nov 2019

HAL is a multi-disciplinary open access archive for the deposit and dissemination of scientific research documents, whether they are published or not. The documents may come from teaching and research institutions in France or abroad, or from public or private research centers.
L'archive ouverte pluridisciplinaire HAL, est destinée au dépôt et à la diffusion de documents scientifiques de niveau recherche, publiés ou non, émanant des établissements d'enseignement et de recherche français ou étrangers, des laboratoires publics ou privés. 


\section{Hybrid metallic ion-exchanged waveguides for SPR biological sensing}

S. de Bonnault, Davide Bucci, Pierre-Jean Zermatten, Paul G. Charette, J. E. Broquin

S. de Bonnault, Davide Bucci, Pierre-Jean Zermatten, Paul G. Charette, J. E. Broquin, "Hybrid metallic ion-exchanged waveguides for SPR biological sensing," Proc. SPIE 9365, Integrated Optics: Devices, Materials, and Technologies XIX, 93650D (27 February 2015); doi: 10.1117/12.2077648 


\title{
Hybrid metallic ion-exchanged waveguides for SPR biological sensing
}

\author{
S. de Bonnault ${ }^{\mathrm{a}}$, D. Bucci ${ }^{\mathrm{a}}$, P.J. Zermatten ${ }^{\mathrm{b}}$, P. G. Charette ${ }^{\mathrm{b}}$, J.E. Broquin ${ }^{\mathrm{a}}$ \\ ${ }^{a}$ Univ. Grenoble Alpes, CNRS, IMEP-LAHC, F-38000 Grenoble, France; ${ }^{b}$ Université de \\ Sherbrooke, Bat. P2- 3IT, 3000 blvd de l'Université, J1K OA5 Sherbrooke (Qc).
}

\begin{abstract}
Glass substrates have been used for decades to create biosensors due to their biocompatibility, low thermal conductivity, and limited fluorescence. Among the different types of sensors, those based on surface plasmon resonance (SPR) allow exploitation of the sensing lightwave at the vicinity of the sensor surface where small entities such as DNA or proteins are located. In this paper, ion-exchanged waveguides and SPR are combined to create a multianalyte optical sensor integrated onto glass. First the principle of operation is introduced, then the theoretical analysis and design of the sensing element. Simulations have been carried out using the Aperiodic Fourier Modal Method (AFMM) and a custom software that handles ion-exchange index-profiles. Fabrication and characterization processes are also presented. Finally the first experimental spectra are displayed and discussed. The sensor presents a bulk sensibility of 5000nm/RIU.
\end{abstract}

Keywords: Biosensors, surface plasmon resonance, ion exchanged waveguides, multi analyte detection

\section{INTRODUCTION}

Integrated biosensors have been investigated for a few decades because of the numerous advantages they have concerning detection of chemical and biological entities. Indeed, cumbersome and expensive machineries can be nowadays replaced by biochips which reduce the number of steps and the quantities of reagents in the detection procedure ${ }^{1}$. Among the many different technologies that have been proposed, glass integrated optics is very popular in biology because it yields good sensitivities as well as easy implementations of multianalyte detection. More and more of these devices are including the light source and part of the signal processing unit in addition to the detection zone ${ }^{2}$. Such devices are self-sufficient and thus extremely useful for rapid and accurate studies. Using glass as a substrate also brings biocompatibility and low optical losses. Ion-exchange is a technique which enables one to locally increase the refractive index of the glass substrate in order to create a waveguide. This increase is brought by the insertion of ions in the glass matrix when the substrate is heated to a certain temperature. This method has already been used in biosensing as it enables, in addition to the numerous advantages of glass, the integration of efficient optical functions like interferometers or monochromators ${ }^{3}$. To further improve sensitivity, integrated optics can be combined with Surface Plasmon Resonance (SPR), which is a well-known label-free method in the biomedical field and particularly adapted to small entities detection ${ }^{4}$. A plasmon is a solution of Maxwell's equations which exists at the interface between a metal and a dielectric only if the real part of the permittivity of the metal $\varepsilon_{\mathrm{m}}$ is negative. As $\varepsilon_{\mathrm{m}}$ depends on the wavelength, this condition is fulfilled in the visible and VIS-IR spectrum only for specific metals such as Gold and Silver. When excited, its presence can be assessed by a resonance, whose existing condition depends on the nature of the dielectric, in this case the analyte. Because the plasmon is localized in the vicinity of the metal, it enables detection of small entities (under $1 \mu \mathrm{m}$ in size).

Biodetection combining integrated optics and SPR has been studied since the 90's, often presenting a channel waveguide with a single central gold layer ${ }^{5,6}$. However, most biological and chemical studies request several measurements and require investigating different entities in parallel. Some studies have proposed multianalyte system based on a prism ${ }^{7} \mathrm{or}$ waveguides ${ }^{8}$. Of these studies, almost none of them propose an integrated version that is easily realizable and extendable to more than two detections at a time.

This paper presents the design, fabrication and characterization of the building block of a multianalyte system combining SPR with integrated optics on glass while providing a spectral detection. The principle of operation is first presented, followed by the design of the single detection zone. The fabrication and characterization are then exposed and finally the experimental results are displayed and discussed. The article closes with some perspectives and a conclusion.

Integrated Optics: Devices, Materials, and Technologies XIX, edited by Jean-Emmanuel Broquin,

Gualtiero Nunzi Conti, Proc. of SPIE Vol 9365, 93650D · (c) 2015 SPIE

CCC code: $0277-786 \mathrm{X} / 15 / \$ 18 \cdot$ doi: $10.1117 / 12.2077648$

Proc. of SPIE Vol. 9365 93650D-1 


\section{PRINCIPLE OF OPERATION}

The proposed multianalyte device shown in Fig. 1 consists of a single waveguide on which several gold blocks are deposited to define the different detection zones, including an additional block for the reference measurement. A broadband source is used to scan the detection zone and collect the resonance spectra. Having the blocks in series instead of parallel allows a single spectrum recording unit.

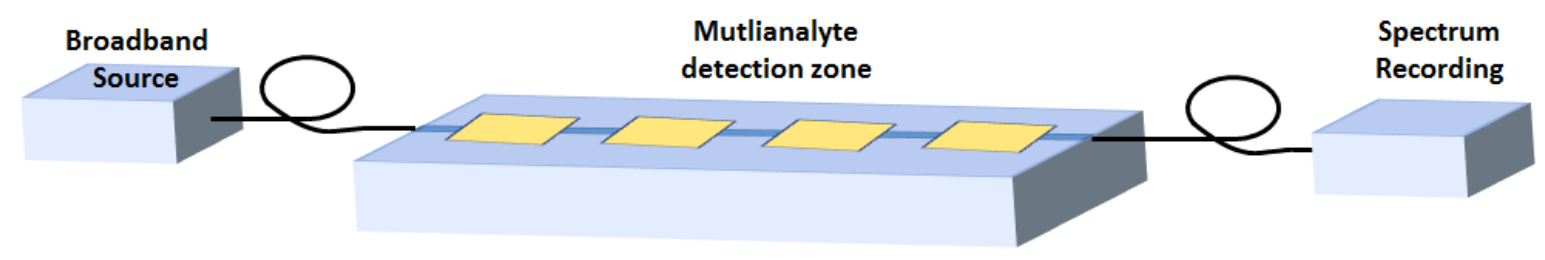

Figure 1. Structure of the integrated multianalyte device

In order to properly design the multianalyte detection zone, it is necessary to first study a single zone as indicated in Fig. 2, consisting of an ion-exchanged waveguide on glass and a thin layer of gold deposited on top of it.

Each zone is divided into three parts: injection, detection, and collection. The injection region guides the optical signal from the broadband source into the detection zone, where the gold layer is deposited. Once the optical signal passes through the detection zone, a plasmon mode can be excited depending on the injected wavelength, creating high losses in the waveguide mode. The spectral response is then measured at the output of the collection section.

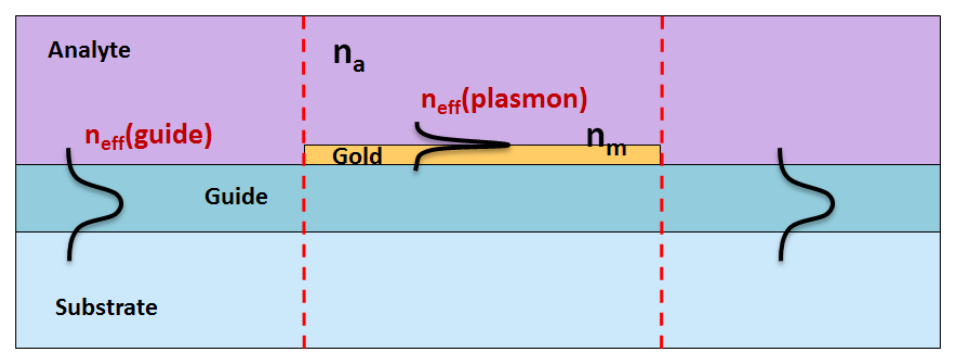

Figure 2. Structure of the integrated optical waveguide combined with SPR.

The plasmon is a surface mode characterized by its propagation constant $\beta_{\mathrm{p}}$, depending on the wavelength $\lambda$ and the permittivity of the metal $\varepsilon_{\mathrm{m}}$ and the dielectric $\varepsilon_{\mathrm{a}} \cdot{ }^{9}$

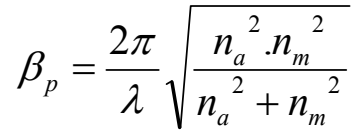

The excitation of the plasmon by a guided mode in the second zone can be described at first approximation by a coupling mode theory which describes the energy transfer between two structures with overlapping modes. This model shows that the maximum energy is transferred from one mode to the other if the propagation constants (or effective indices) of both overlapping modes are equal (phase matching condition). The fulfillment of this condition triggers a resonance. It must be noted here that the coupling theory usually applies to waveguides separated by a low refractive index, and whose modes are not as close as the modes from the plasmonic structure. It describes a weak coupling theory, which is not exactly the case here but can be still considered as a good approximation.

As the effective indices $n_{\text {eff }}$ of the guided mode and the plasmon are related to the wavelength $\lambda$, the resonance can be found by plotting the effective indices for each mode of the two separate structures. The choice of the structure decomposition and the corresponding $\mathrm{n}_{\mathrm{eff}}=\mathrm{f}(\lambda)$ curves are presented in Fig. 3 (left). The gold is present in each substructure as it corresponds to the connecting medium between both modes. 

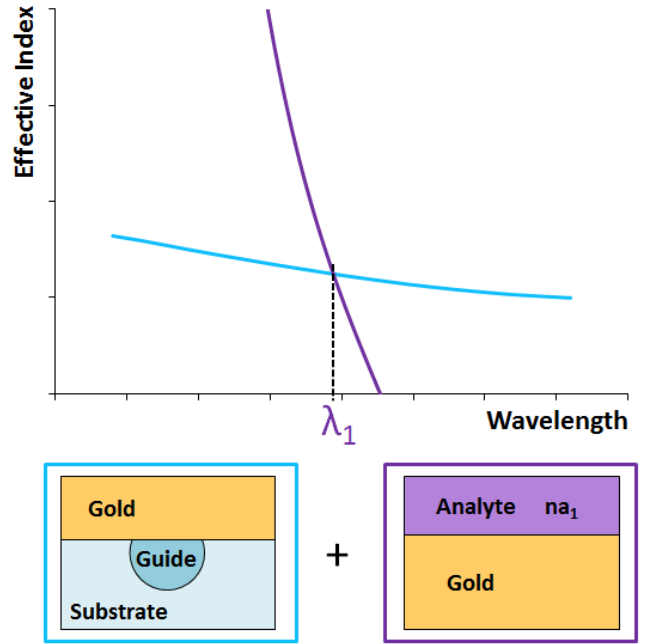
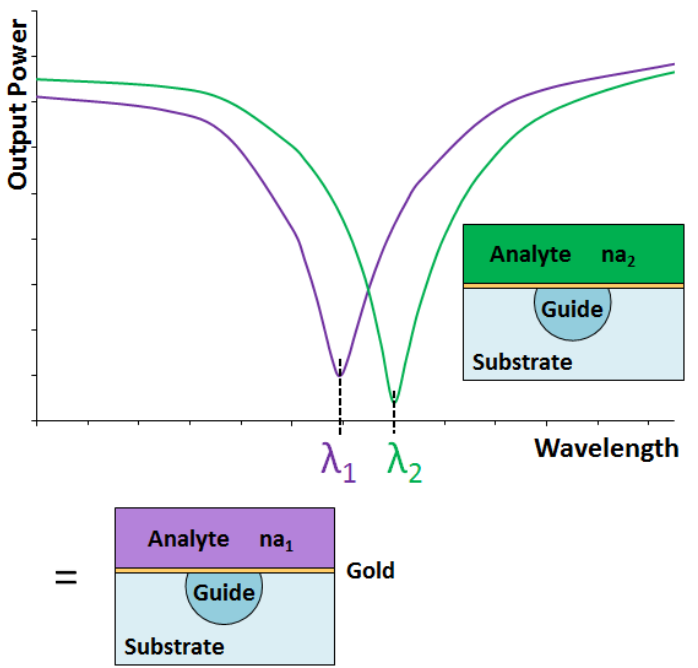

Figure 3. Structures used for the phase matching technique and corresponding curves.

Once the resonance is triggered (Fig. 3 Right) for a wavelength $\lambda_{1}$, if the analyte refractive index varies from $\mathrm{na}_{1}$ to $\mathrm{na}_{2}$, the resonance will be shifted from $\lambda_{1}$ to $\lambda_{2}$, making it the basis of SPR detection.

\section{DEVICE DESIGN}

\subsection{Modeling the waveguide}

The graded index profile of the exchanged waveguide is simulated with a finite difference based software which solves the diffusion equation ${ }^{10}$ presented in (2). First the concentration profile of the doping ions is calculated for a given exchange time $t$ through a diffusion aperture $\mathrm{W}$. The index profile is then extracted with the linear relation (3) with $\mathrm{n}_{\text {sub }}$ the substrate index and $\Delta \mathrm{n}_{\max }$ the maximum difference between the substrate and the core refractive indices.

$$
\begin{gathered}
\frac{\partial c}{\partial t}=\nabla(\widetilde{D}(c) \nabla c) \\
n(x, y)=n_{\text {sub }}+\Delta n_{\text {max }} c(x, y)
\end{gathered}
$$

The refractive index map is then put in a finite difference mode solver in order to obtain the field profile and the effective index of the fundamental mode as a function of the wavelength.

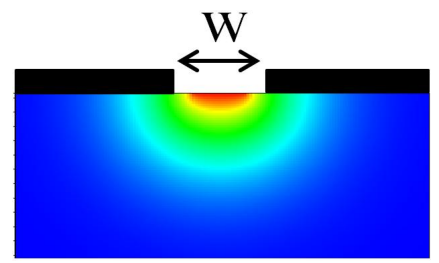

Figure 4. Typical index profile of a surface exchanged waveguide.

\subsection{Designing the waveguide}

The design of the waveguide is based on one criterion: it must permit analyte detection for refractive indices between 1.33 and 1.43 to provide further multianalyte detection. The selected substrate is a borosilicate glass (BF33 from Shott) whose refractive index is approximately 1.465 in the near-infrared range. The substrate presents the advantages of high chemical and mechanical resistance while maintaining a low-cost. The exchange is directed with $\mathrm{Ag}+$ ions replacing the $\mathrm{Na}+$ ions present in the glass matrix. The effective index of the waveguide will therefore vary between 1.465 and the highest value possible for the graded-index core which is 1.485 . The phase-matching condition is used to design the 
waveguide parameters $t$ and W. Fig. 5 presents the results of simulation. The effective indices of the plasmons for analyte refractive indices form 1.33 to 1.43 are plotted over the wavelength.

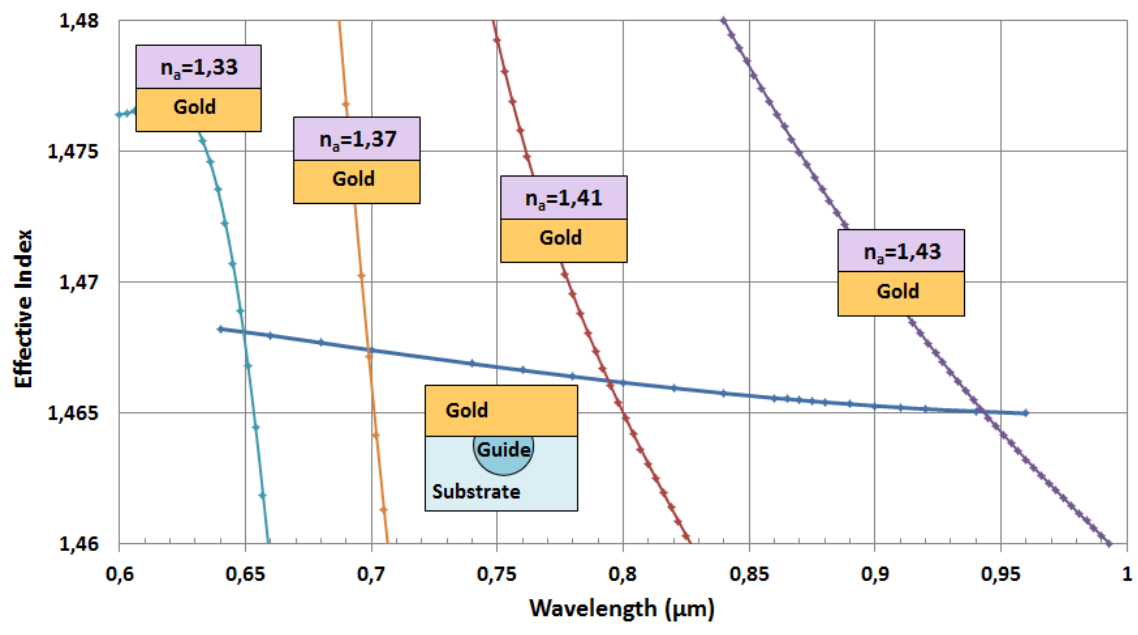

Figure 5. Phase matching technique to design the waveguide: effective indices as function of $\lambda$.

The selected 2D waveguide permits to verify the condition, as its effective index equals that of the desired plasmons for different wavelengths. It corresponds to an exchange time t of 30 min and an opening $\mathrm{W}$ of $0.8 \mu \mathrm{m}$.

\subsection{Modeling sensor response}

The sensor response is obtained by calculating the transmission of the structure presented in Fig. 6 . This calculation requires to consider now the hybrid modes (or supermodes) of the structure. The injected mode couples power at the interface (a) into the three main supermodes $\Psi_{i}$ created with the excitation of the plasmon. These modes then propagate over a distance $\mathrm{L}$ and recouple at the interface (b) into the output mode $\Phi_{\text {OUT }}$.

(a)

(b)

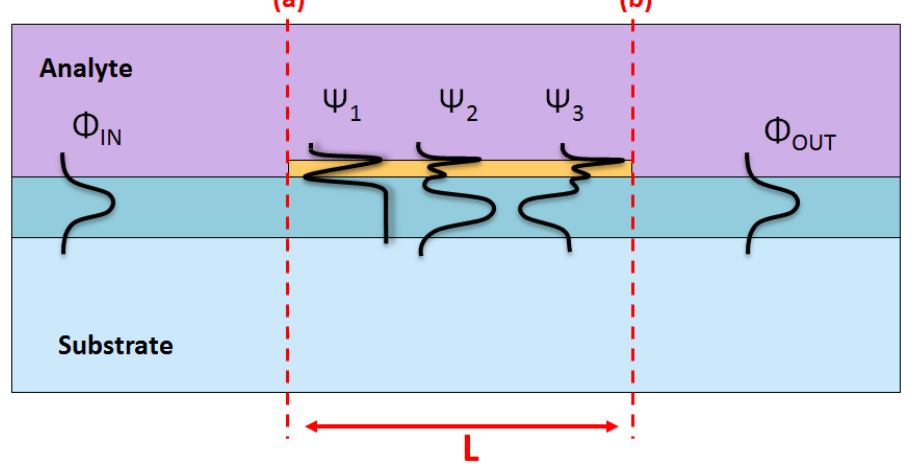

Figure 6. Structure for the transmission calculation.

The expression of the transmission can be written as follows:

$$
T_{\text {OUT }}(\lambda)=\left|\sum_{i=0}^{2} C_{i} e^{j \beta_{i} L}\right|^{2}(\lambda)
$$

Where $C_{i}$ is the coupling coefficient related to the mode $\Psi_{\mathrm{i}}$, L is the gold length to be determined and $\beta_{\mathrm{i}}$ is the complex propagation constant of the mode $i$ which contains the real and imaginary parts of each mode's effective index.

The study logic is here for one wavelength $\lambda$ to find the mode profiles and their effective indices, then calculate the coupling coefficient and finally the transmission $\mathrm{T}_{\text {OUT }}$. Several iterations over the wavelength enable at last to obtain the transmission spectrum for the device. 
The modal analysis of the detection zone is carried out with a custom full-vectorial mode solver based on the Aperiodic Fourier Modal Method (AFMM). This technique, proposed by Lalanne and Silberstein ${ }^{11}$ applies the Rigorous Coupled Wave Method (RCWA) to waveguides, which is normally used to study diffraction gratings. With some refinements this method indicates positive results for SPR structures and more generally, any devices based on waveguides.

The structures can be implemented using the AFMM which outputs the effective index and field profile of any mode it can find.

Refractive indices of gold varying with wavelength are taken from E.D. Palik's Handbook of Optical Constants of Solids ${ }^{12}$. The indices of the analyte $n_{a}$ and the substrate $n_{s}$ are considered constant over $\lambda$, as their variation is negligible compared to the gold one. Fig. 7 shows the field profile as a function of the wavelength for the three main hybrid modes found in the detection zone. The first one, called TM0 is a mode with both a very high effective index and attenuation $\left(>3.10^{4} \mathrm{~dB} / \mathrm{cm}\right)$. Injected light is hardly coupled in this mode which profile is barely overlapping with the injected mode. The resonance will mainly be governed by the two other supermodes TM1 and TM2. Looking at the mode profiles it can be deduced that the injected mode will essentially couple into the TM2 mode at short wavelengths and into the TM1 mode as the wavelength increases and TM2 reaches cut-off.

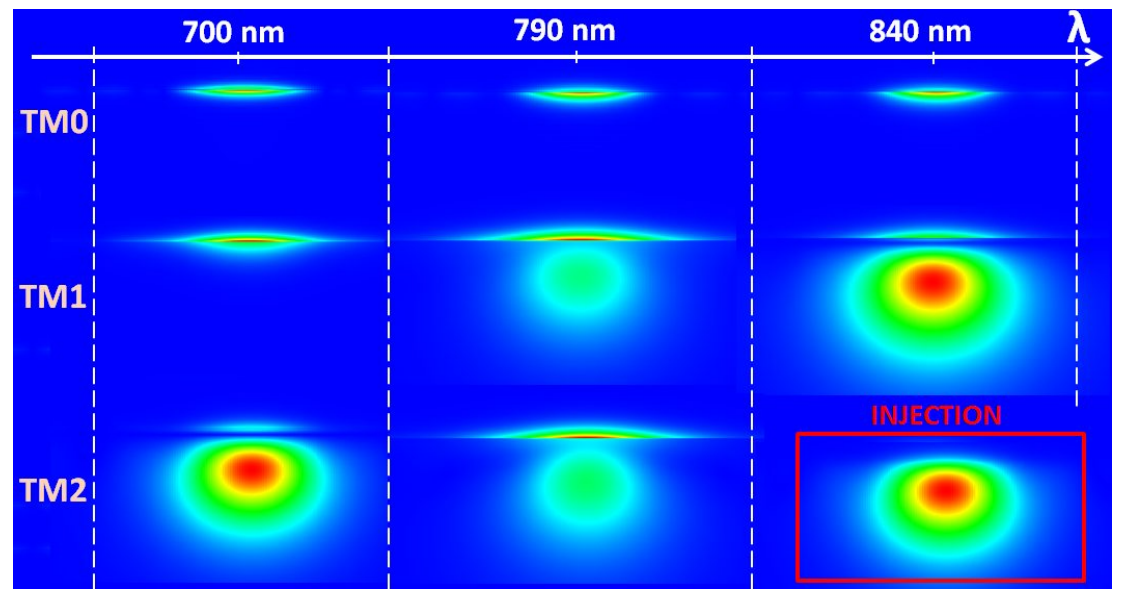

Figure 7. Field profiles of the three main hybrid modes of the detection zone and the injection mode, over the wavelength.

Once the field profiles and effective indices are obtained with the AFMM mode solver, the coupling coefficients have to be calculated. The standard method considers the modes as orthogonal and therefore gives an independent expression for each coefficient (Eq. (5)) ${ }^{13}$.

$$
C_{i}^{O r t h}=\frac{\left(\iint E_{y 0} H_{x i}^{*} d x d y\right)\left(\iint E_{y i} H_{x 0}^{*} d x d y\right)}{\left(\iint E_{y i} H_{x i}^{*} d x d y\right)\left(\iint E_{y 0} H_{x 0}^{*} d x d y\right)}
$$

However, as the metal brings high losses, the modes are actually non orthogonal. In that case, the coefficients are related and their expressions have to be extracted from a linear system of three equations presented in Eq. (6). The subscript "0" corresponds to the injection mode and " 1 ", "2" and " 3 " to the three TM modes.

$$
\left\{\begin{array}{l}
\iint E_{y 0} H_{x 1}^{*} d x d y=\sum_{j=0}^{2} \iint C_{j}^{\text {NonOrth }} \cdot\left(E_{y j} H_{x 1}^{*} d x d y\right) \\
\iint E_{y 0} H_{x 2}^{*} d x d y=\sum_{j=0}^{2} \iint C_{j}^{\text {NonOrth }} \cdot\left(E_{y j} H_{x 2}^{*} d x d y\right) \\
\iint E_{y 0} H_{x 3}^{*} d x d y=\sum_{j=0}^{2} \iint C_{j}^{\text {NonOrth }} \cdot\left(E_{y j} H_{x 3}^{*} d x d y\right)
\end{array}\right.
$$

These values have then to be determined for several wavelengths in order to get the final response. 
Fig. 8 presents the transmission with an analyte index $n_{a}=1.41$, and different length $\mathrm{L}(250 \mu \mathrm{m}, 500 \mu \mathrm{m}$ and $1 \mathrm{~mm})$. The transmission is calculated with Eq. (4) with the $C_{i}$ calculated with the linear system in Eq. (6).

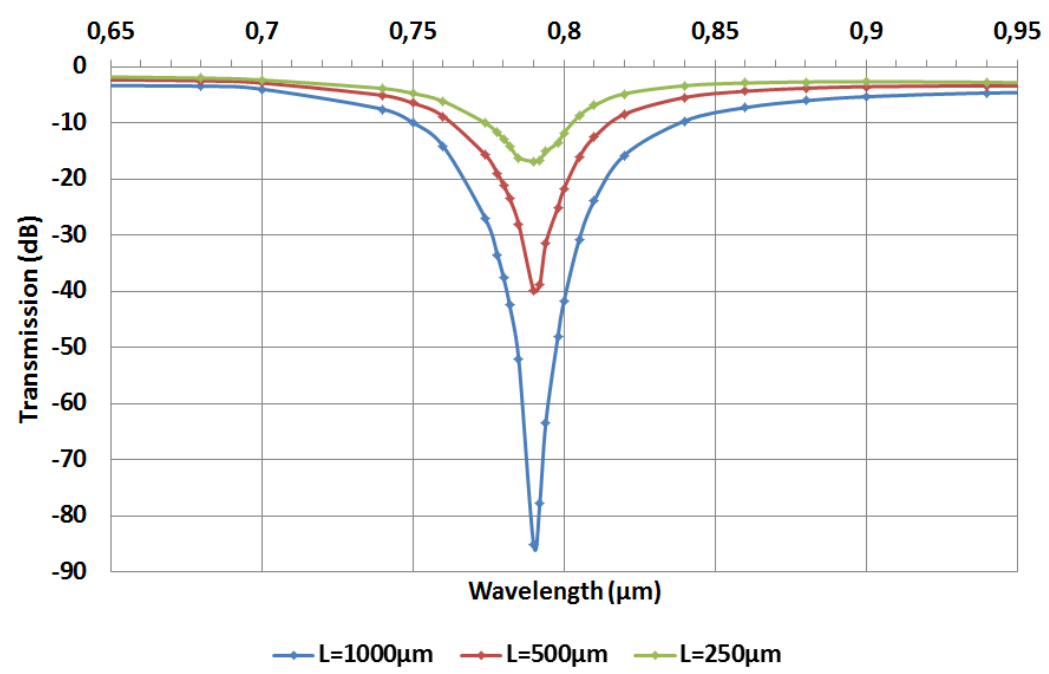

Figure 8. Result of simulation with $\mathrm{W}=0.8 \mu \mathrm{m}, \mathrm{t}=30 \mathrm{~min}, \mathrm{n}_{\mathrm{s}}=1.465, \mathrm{n}_{\mathrm{a}}=1.41$ and different $\mathrm{L}$.

It can be seen that the resonance is deeper and larger as the gold length increases. For $\mathrm{L}=1000 \mu \mathrm{m}$, the minimum transmission occurs for $\lambda=790 \mathrm{~nm}$. To ensure a deep enough resonance, the experimental length $\mathrm{L}$ is fixed to $1100 \mu \mathrm{m}$.

\subsection{Device sensitivity}

The device sensitivity is calculated by measuring the resonance wavelength shift when the analyte refractive index varies from 0.01 RIU. Fig. 9 shows the theoretical sensitivity for the refractive index increasing from 1.41 to 1.42.

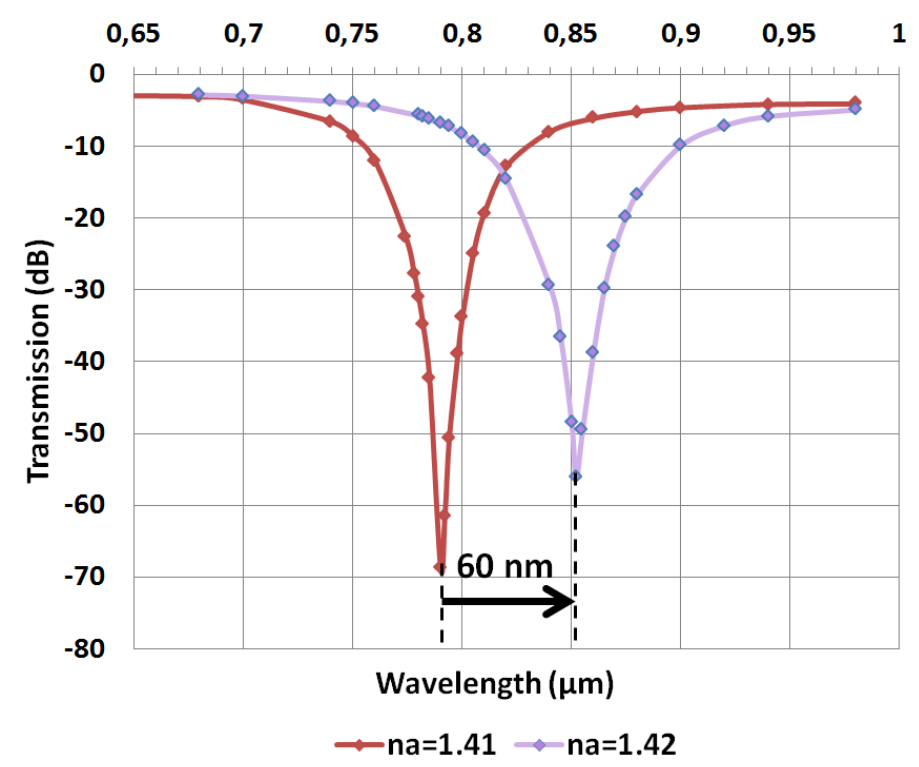

Figure 9. Shift of the resonance for two different analyte refractive indices (1.41 and 1.42).

The sensitivity corresponds to difference between the resonances wavelength taken at the minimum transmission of each curve. It is equal to $790 \mathrm{~nm}$ for a refractive index of 1.41 and $850 \mathrm{~nm}$ for a refractive index of 1.42 , that is to say a $60 \mathrm{~nm}$ shift for a $0.01 \mathrm{RIU}$ difference. The value obtained is $6000 \mathrm{~nm} / \mathrm{RIU}$, which is close to the values presented in the literature ${ }^{14}$. 


\section{FABRICATION AND CHARACTERIZATION}

\subsection{Fabrication}

The BF33 contains $4.5 \%$ of sodium oxide. The fabrication process presented on Fig. 10 begins with a thin aluminum oxide layer deposited onto the cleaned substrate.

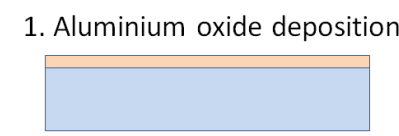

2. Photolithography

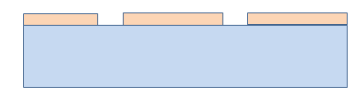

3. Exchange

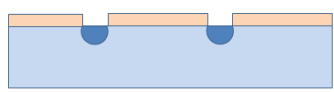

4. Removal of $\mathrm{Al}_{2} \mathrm{O}_{3}$ layer
5. Chromium deposition

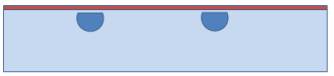

6. Gold deposition

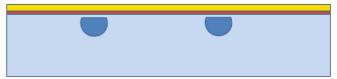

7. Photolithography

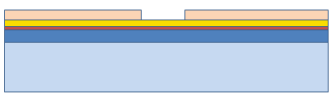

8. Metal etching
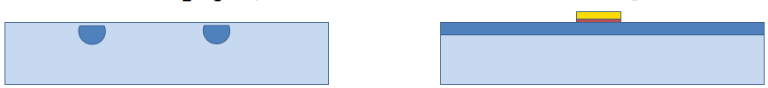

Figure 10. Process for the device fabrication

Following this, a photolithography is performed which enables the opening of diffusion apertures $\mathrm{W}$ in the deposited layer with sizes ranging from $0.5 \mu \mathrm{m}$ to $10 \mu \mathrm{m}$. The substrate is afterwards immersed in a bath of $80 \%$ silver nitrate and $20 \%$ sodium nitrate at $353^{\circ} \mathrm{C}$ for 30 minutes. During this period, sodium ions migrate through the apertures in the salt and silver ions replace them in the glass matrix to form the waveguide. Gold is then sputtered onto the entire surface of the substrate over a fine layer of chromium used to promote adhesion of the metal on glass. Another photolithography step is performed in order to pattern the thin gold film. The strip is obtained using a chemical etching process. Finally the wafer is diced and its facets are polished in order to obtain the chip shown in Fig. 11.

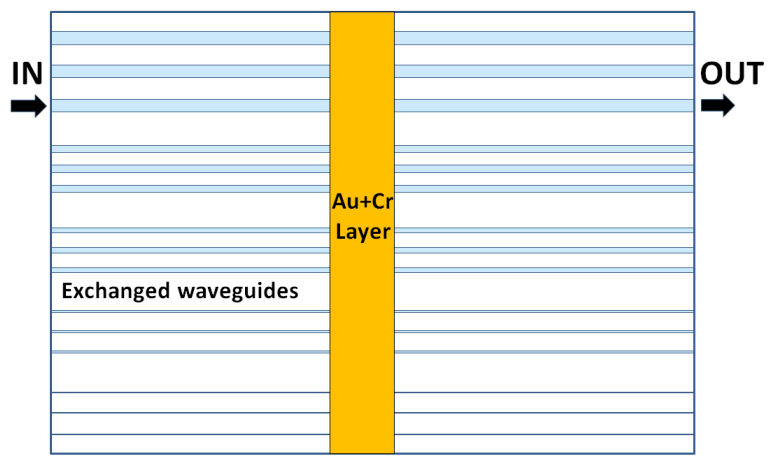

Figure 11. Layout of the fabricated structure.

\subsection{Characterization}

The optical bench shown in Fig. 12 has been used to characterize the sensor. It consists of a supercontinuum source (Leukos SA) providing a broadband light input ranging between $400 \mathrm{~nm}$ and $1600 \mathrm{~nm}$. The light is injected into the device using a HI 1060 fiber and a micro-positioning stage. The output of the waveguide is imaged through a microscope objective on a spectrometer. A polarizer is inserted between the microscope objective and the OSA in order to select either the Transverse Magnetic (TM) or Transverse Electric (TE) spectra. As the resonance occurs only in TM, it is interesting to compare both spectra in real time in order to quickly identify the plasmonic resonance. 


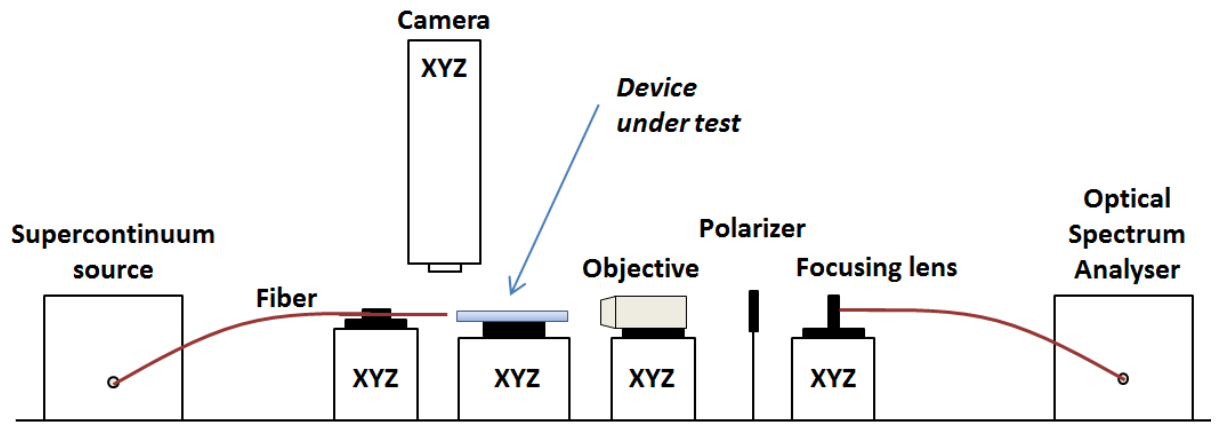

Figure 12. Optical bench used to characterize the device.

The device is tested using drops of different analytes, with refractive index ranging from 1.33 to 1.45. For each analyte, TE and TM spectra are recorded and normalized with baseline TE and TM spectra of the device in air, with a resolution of $5 \mathrm{~nm}$.

\section{RESULTS AND DISCUSSION}

Fig. 13(left) shows the first experimental resonance observed for refractive index of 1.41.
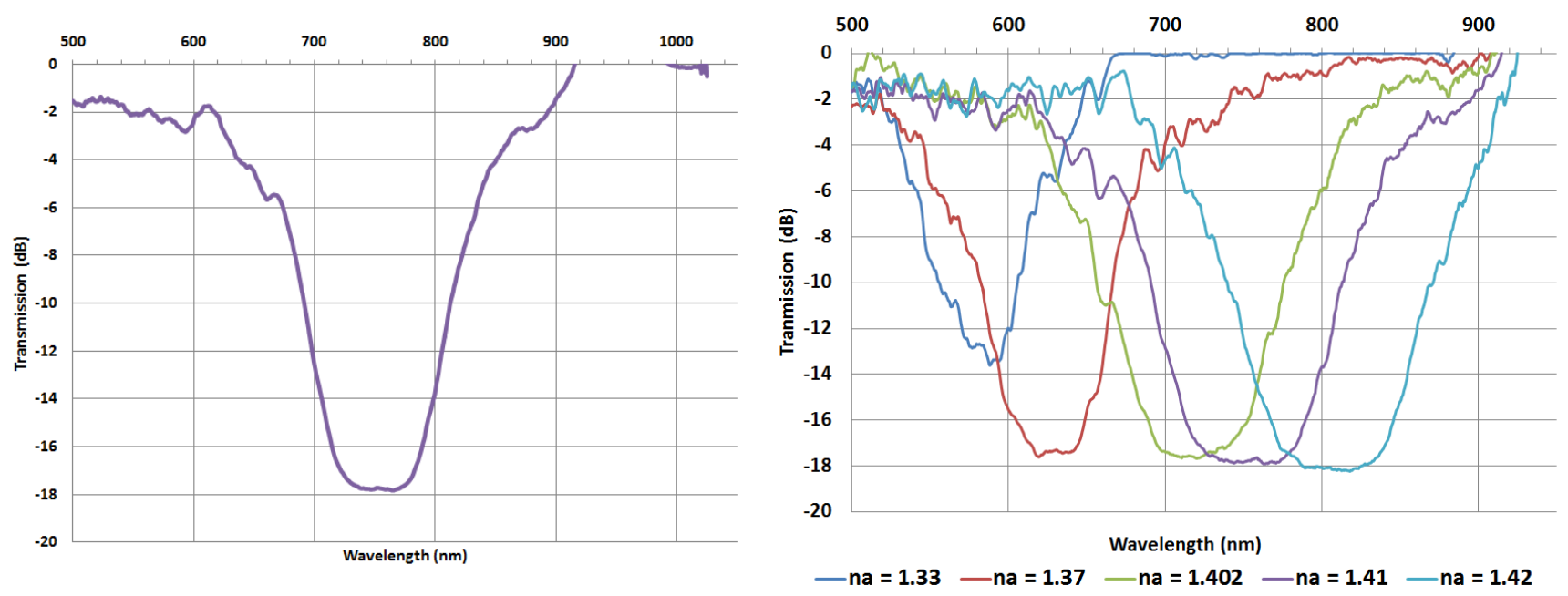

Figure 13. Experimental result for $\mathrm{n}_{\mathrm{a}}=1.41$ (left) and for $\mathrm{n}_{\mathrm{a}}=1.33$ to 1.42 (right).

The experimental curve presents an absorption peak of $16 \mathrm{~dB}$ with an uncertainty about $1 \mathrm{~dB}$ due to measurements. The plateau at $-18 \mathrm{~dB}$ is due to the OSA noise level which limits the dynamic of the measurement.

Fig. 13(right) presents another set of measurements on the same waveguide with liquids of refractive indices ranging from 1.33 to 1.42 , highlighting the shift of the resonance when the analyte changes. These results demonstrate the multianalyte potential of the structure for further implementation. As the waveguide length is over $3 \mathrm{~cm}$ and the wavelength span is large enough, it will be possible to deposit several gold strips and make parallel measurements in different refractive index areas. Moreover an experimental sensitivity can be determined by measuring at a fixed transmission the wavelength shift between two spectra obtained for different analyte refractive indices. It is equal to $4800 \mathrm{~nm} / \mathrm{RIU}$ if the calculation is made for refractive indices equal to 1.41 and 1.42 .

The comparison between the experimental and theoretical results is introduced in Fig. 14. 


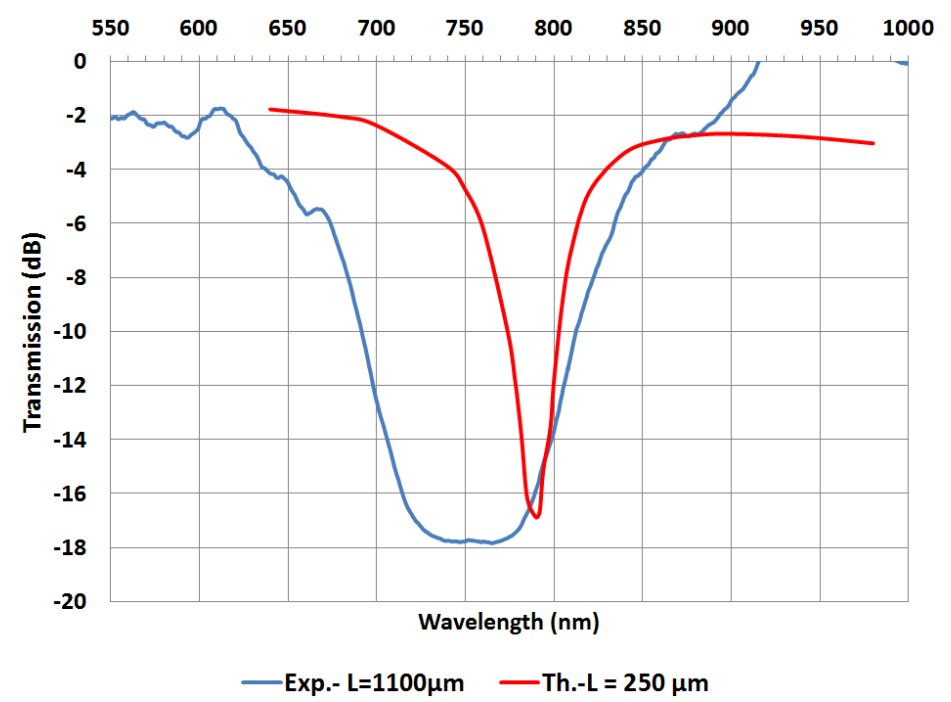

Figure 14. Comparison between the experimental resonance and the simulated resonance for $\mathrm{n}_{\mathrm{a}}=1.41$.

Although no conclusion can be drawn for the width of the measured resonance because of the signal saturation, its position is clearly blue-shifted about $40 \mathrm{~nm}$. The first explanation comes from the fact that the broadening of the waveguide through the photolithographic steps has not been taken into account during the simulation. The waveguide is wider and thus its effective index higher, leading to a blue-shift.

\section{CONCLUSION}

In this article an efficient method for designing an integrated optical device combined with SPR is presented. It has been demonstrated that the approach based on the 2D diffused guide is a good model for creating a resonance for a desired analyte refractive index range. The realized single detection device has a wavelength operating range between $500 \mathrm{~nm}$ and $900 \mathrm{~nm}$ and a can detect refractive indices from 1.33 to 1.43 . Its sensitivity is about $5000 \mathrm{~nm} / \mathrm{RIU}$ around a refractive index of 1.41 .

Preliminary results are encouraging and the next short-term step will therefore be to optimize the optical benches and test the device with biological material. The mid-term objective will then be to fabricate the multianalyte detection zone. In the long run, the multianalyte sensor could be implemented with other detection methods such as microcalorimetry or fluorescence to cross-check or complete the information about the studied chemical or biological reaction.

\section{REFERENCES}

[1] Lambeck, P.V., "Integrated optical sensors for the chemical domain.” Meas. Sci. Technol. 17(8), 93-116, (2006).

[2] Balslev, S., Jorgensen, M., Bilenberg, B., Mogensen, K. B., Snakenborg, D., Geschke, Kristensen, A. "Lab-ona-chip with integrated optical transducers". Lab on a Chip 6(2), 213-217 (2006).

[3] Broquin, J-E, "Glass integrated optics: state of the art and position toward other technologies." Proc. SPIE, 6475, 647507-647513 (2007).

[4] Liedberg, B., Nylander, C., and Lundström, I., "Biosensing with surface plasmon resonance-how it all started." Biosens. Bioelectron. 10, i-ix, (1995).

[5] Hugon, O., Trouillet, A., Gagnaire, H., and Tobis, P., "An integrated optic hydrogen sensor based on SPR on palladium." Sensors and Actuators 74, 168-172, (2001).

[6] Harris, R. D., Luff, B. J., Wilkinson, J. S., Piehler, J., Brecht, A., Gauglitz, G., and Abuknesha, R., "Integrated optical surface plasmon resonance immunoprobe for simazine detection." Biosens. Bioelectron. 14(4), 377-86, (1999).

[7] Homola, J., Lu, H. B., Nenninger, G. G., Dostálek, J., and Yee, S. S., "A novel multichannel surface plasmon resonance biosensor." Sensors Actuators B Chem. 76, 403-410, (2001). 
[8] Akowuah, E. K., Gorman, T., Haxha, S., and Oliver, J. V., "Dual channel planar waveguide surface plasmon resonance biosensor for an aqueous environment." Opt. Express 18(23), 24412-24422, (2010).

[9] Burke, J.J., Stegeman, G. I., and Tamir, T., "Surface-polariton-like waves guided by thin, lossy metal films." Phys. Rev. B 33(8), 5186-5201, (1986).

[10] Tervonen, A., West, B. R. and Honkanen, S. "Ion-exchanged glass waveguide technology: a review." Opt. Eng. 50(7), 071107 1-071107 15, (2011).

[11] Lalanne P., and Silberstein, E., "Fourier-modal methods applied to waveguide computational problems." Opt. Lett. 25(15), 1092-4, (2000).

[12] Palik, E.D., [Handbook of Optical Constants of Solids], Acaddemic Press (1998)

[13] Čtyroký, J., Homola, J., Lambeck, P. V., Musa, S., Hoekstra, H. J. W. M., Harris, R. D., Wilkinson, J. S., Usievich, B., and Lyndin, N. M., "Theory and modelling of optical waveguide sensors utilising surface plasmon resonance." Sensors Actuators B Chem. 54, 66-73, (1999).

[14] Dostalek, J., Ctyroky, J., Homola, J., Brynda, E., Skalsky, J., Nekvindova, P., Spirkova, J., Skvor, J., and Schröfel, J., "Surface plasmon resonance biosensor based on integrated optical waveguide." Sensors Actuators B 76, 8-12, (2001). 